\title{
IDENTIFICAÇÃO DE COMPORTAMENTOS ALIMENTARES, TEMPO EM FRENTE À TELEVISÃO E ATIVIDADES FÍSICAS DE ADOLESCENTES
}

\section{Identification of adolescents' eating behaviors, time spent in front of television and physical activity}

\section{Identificación de conductas alimentarias, tiempo delante de la televisión y actividades físicas de adolescentes}

\author{
Francielle Klouck \\ Universidade do Extremo Sul Catarinense - UNESC - Criciúma (SC) - Brasil \\ Joni Márcio de Farias \\ Universidade do Extremo Sul Catarinense - UNESC - Criciúma (SC) - Brasil

\section{Eduarda Valim Pereira} \\ Universidade do Extremo Sul Catarinense - UNESC - Criciúma (SC) - Brasil
}

\section{Vanise dos Santos Ferreira Viero}

Universidade do Extremo Sul Catarinense - UNESC - Criciúma (SC) - Brasil

\section{RESUMO}

Objetivo: Identificar os comportamentos relacionados aos hábitos alimentares, às atividades físicas, ao tempo em frente à tela de televisão (TV) e ao deslocamento ativo de adolescentes. Métodos: Estudo transversal, realizado em escola estadual do município de Praia Grande, Santa Catarina, Brasil. A amostra foi composta por 86 adolescentes, de ambos os sexos, com idade entre 14 a 19 anos, matriculados no ensino médio. O instrumento de pesquisa utilizado foi elaborado a partir da composição de indicadores validados, composto por 30 perguntas objetivas sobre dados sociodemográficos, hábitos alimentares e de atividades físicas, deslocamento ativo e tempo em frente à tela. Os dados foram tabulados no SPSS, versão 21.0 , avaliando frequência absoluta, relativa, média, desvio padrão, teste $t$ de Student e correlação "r" de Pearson, adotando-se o nível de significância de 95\% ( $p \leq 0,05$ ). Resultados: Dos 86 adolescentes, 40,7\% relataram que raramente consomem uma dieta balanceada, 53,5\% têm como lazer preferido as atividades físicas, 33,7\% deslocam-se a pé para a escola e 31,4\% utilizam a bicicleta. Referente ao tempo em frente à tela, meninos têm maior exposição ( $8 \mathrm{~h}$ e $10 \mathrm{~h}$ ) do que as meninas ( $5 \mathrm{~h}$ e $6 \mathrm{~h}$ ) em dias de semana e finais de semana, respectivamente. Conclusão: As características geográficas, as atividades físicas habituais e a segurança pública contribuem para um estilo de vida saudável no contra turno, exceto nos hábitos alimentares, indicando a necessidade do estabelecimento de estratégias que estimulem nesses jovens a adoção de hábitos saudáveis de vida.

Descritores: Adolescente; Estilo de Vida; Saúde Coletiva.

\begin{abstract}
Objective: To identify adolescents' behaviors related to eating habits, physical activity and time spent in front of television (TV) screens. Methods: This is a cross-sectional study carried out in a state-run school in the municipality of Praia Grande, Santa Catarina, Brazil. The sample consisted of 86 adolescents, of both sexes, aged 14 to 19 years, enrolled in high school. The research instrument used was based on validated indicators and consisted of 30 closed-ended questions about sociodemographic data, eating and physical activity habits, active behaviors and time in front of screens. The data were tabulated in SPSS version 21.0 and analyzed using absolute and relative frequencies, mean, standard deviation, Student's $t$ test and Person's " $r$ " correlation with a significance level set at $95 \%$ ( $p \leq 0.05$ ). Results: Of the 86 adolescents, $40.7 \%$ reported rarely following a balanced diet, $53.5 \%$ reported physical activity as their favorite leisure activities, $33.7 \%$ walked to school and $31.4 \%$ used the bicycle. As for time in front of screens, boys presented greater exposure (8h and 10h) than girls (5h and 6h) on weekdays and weekends, respectively. Conclusion: Geographic characteristics, habitual physical activities and public safety contribute to a healthy lifestyle, except for eating habits, thus indicating the need to establish strategies that encourage these young people to adopt healthy life habits.
\end{abstract}

Descriptors: Adolescents; Life Style; Public Health. 


\section{RESUMEN}

Objetivo: Identificar las conductas relacionadas con los hábitos alimentarios, las actividades físicas, el tiempo delante de la televisión (TV) y el desplazamiento activo de adolescentes. Métodos: Estudio transversal realizado en una escuela estadual del municipio de Praia Grande, Santa Catarina, Brasil. La muestra fue de 86 adolescentes de ambos sexos, de edad entre 14 y 19 años de la educación secundaria. El instrumento de investigación utilizado ha sido desarrollado a partir de la composición de indicadores validados con 30 preguntas objetivas sobre los datos sociodemográficos, los hábitos alimentarios y las actividades físicas, el desplazamiento activo y el tiempo delante de la tele. Los datos fueron tabulados en el SPSS versión 21.0 y evaluado la frecuencia absoluta, la relativa, la media, la desviación típica, la prueba t de Student y la correlación " $r$ " de Pearson adoptandose el nivel de significación del 95\% ( $p \leq 0,05)$. Resultados: De entre los 86 adolescentes, el 40,7\% relataron que casi nunca consumen una dieta balanceada, el 53,5\% prefieren el ocio con actividades físicas, el 33,7\% se desplazan caminando para la escuela y el 31,4\% utilizan la bicicleta. Respecto el tiempo delante de la tele, los niños tiene más exposición (8h y 10h) que las niñas (5h y 6h) entre semana y fines de semana, respectivamente. Conclusión: Las características geográficas, las actividades físicas habituales y la seguridad pública contribuyen para un estilo de vida saludable en el contra turno, excepto para los hábitos alimentarios lo que indica la necesidad de estrategias que estimulen eses jóvenes para la adopción de hábitos de vida saludables.

Descriptores: Adolescente; Estilo de Vida; Salud Pública.

\section{INTRODUÇÃO}

As fases da infância e da adolescência são essenciais para adesão aos hábitos saudáveis e a um estilo de vida ativo, prospectando que esses comportamentos sejam transferidos para a vida adulta( ${ }^{(1,2)}$. No entanto, as evidências demonstram que o oposto vem ocorrendo, com uma população com maior propensão a comportamentos de risco à saúde e baixos níveis de atividade física( ${ }^{(3)}$.

Os contributos que auxiliam para a diminuição da qualidade de vida e saúde dos adolescentes são: o baixo nível de atividade física ${ }^{(4,5)}$, frequentemente associado ao acúmulo excessivo de gordura corporal; alterações desfavoráveis no perfil dos lipídios sanguíneos; níveis elevados de pressão arterial; diabetes; osteoporose; algumas formas de câncer ${ }^{(6)}$ e a exposição a outros comportamentos de risco à saúde ${ }^{(7)}$.

Ao se analisar de maneira isolada os resultados da PeNSE (2015), que avaliou adolescentes de 13 a 17 anos de todas as capitais brasileiras, a prevalência de adolescentes insuficientemente ativos foi de $60,5 \%$, com ponto de corte de menos de $300 \mathrm{~min} / \mathrm{semana}$ de atividade física. No tempo em frente à tela de televisão (TV), aproximadamente $53,8 \%$ dos adolescentes permanecem mais de duas horas por semana. Já quando indagados sobre a utilização de outros instrumentos de tela, como videogames e computadores ou outra atividade em que permaneçam sentados, $52,9 \%$ relatam ficar mais de três horas por semana ${ }^{(8)}$.

Além dos baixos níveis de atividade física e do comportamento sedentário, os adolescentes consomem, em maior quantidade, alimentos de alto teor calórico e com baixos nutrientes, como os processados e ultraprocessados, quando deveriam ter uma maior ingestão de alimentos saudáveis, como os in natura e minimamente processados ${ }^{(9,10)}$. Um estudo transversal, de base populacional, que analisou os comportamentos alimentares saudáveis e não saudáveis entre adultos jovens, de meia idade e idosos brasileiros, constatou que os adultos jovens apresentaram, em geral, menor frequência de consumo regular de alimentos saudáveis e maior frequência de alimentos não saudáveis quando comparados aos de meia idade e idosos, indicando a necessidade de se estabelecer ações e estratégias para adoção de hábitos alimentares mais saudáveis desde a infância, os quais perdurarão para todas as idades ${ }^{(11)}$.

Esses comportamentos contribuem para o desenvolvimento da obesidade e do sobrepeso, como aponta a última análise da PeNSE de 2015, atestando uma prevalência de $23,7 \%$ de excesso de peso e $7,8 \%$ de obesidade entre os adolescentes brasileiros ${ }^{(8)}$. Diante desse cenário, faz-se necessário o conhecimento dos hábitos dos adolescentes relacionados à prática de atividade física diária e de lazer, do tempo em frente à tela de TV e dos hábitos alimentares, considerando que o conhecimento desses fatores é primordial para o estabelecimento de estratégias que promovam e protejam a saúde desses sujeitos, mobilizando a construção e a incorporação de práticas que visem mudanças comportamentais prejudiciais à saúde, considerando o local e a realidade em que se encontram. Sendo assim, o presente estudo teve como objetivo identificar os comportamentos dos adolescentes relacionados aos hábitos alimentares e de atividades físicas, deslocamento ativo e tempo em frente à tela de televisão (TV). 


\section{MÉTODOS}

O estudo caracterizou-se como transversal, com abordagem quantitativa, realizado em uma escola estadual do município de Praia Grande, município brasileiro localizado no extremo Sul Catarinense, o qual possui uma área territorial de $286,11 \mathrm{~km}^{2}$. O município conta com uma população de 7.270 habitantes e a densidade demográfica é de 25,58 habitantes por $\mathrm{Km}^{2}$. A atividade econômica predominante do município é a agricultura, representada pelo cultivo do arroz, milho, fumo e banana ${ }^{(12)}$.

A população do estudo foi constituída por adolescentes, de ambos os sexos, com idade de 14 a 19 anos, matriculados no ensino médio, nos períodos matutino e noturno. Para participar do estudo, os adolescentes deveriam: estar devidamente matriculados na unidade escolar; cursando o ensino médio ( $1^{\circ}$ ao $3^{\circ}$ ano); ter frequência escolar superior a $75 \%$, inclusive nas aulas de educação física; não apresentar limitações para responder os instrumentos; preencher os instrumentos de maneira adequada e trazer o Termo de Consentimento Livre Esclarecido (TCLE) assinado pelos pais e/ou responsáveis. Foram excluídos da amostra os estudantes que preencheram os instrumentos de maneira inadequada, perfazendo um " $\mathrm{n}$ " amostral de 86 adolescentes.

$O$ instrumento de pesquisa utilizado foi elaborado a partir da composição de indicadores validados e utilizados em outros estudos nacionais, composto por 30 perguntas fechadas, divididas em quatro blocos: 1) Identificação; 2) Hábitos alimentares; 3) Hábitos de atividades físicas; 4) Tempo em frente à tela.

1) Identificação: Foram eleitas questões que identificassem os estudantes quanto a situação econômica, local de moradia, trabalho e percepção do estado geral de saúde.

2) Hábitos alimentares: As questões abordavam quantas vezes na semana/dia ingere líquidos que possuam cafeína, se ingere uma dieta balanceada ( 5 a 12 porções de grãos ou cereais, 5 a 10 porções de frutas e vegetais, 2 a 3 porções de carnes e 2 a 4 porções de leite diariamente) e, durante os últimos 30 dias, se já tomou 5 ou mais doses de bebidas alcoólicas.

3) Percepção dos níveis de atividade física: Abordando questões sobre preferência em atividades de lazer divididas em atividade física (prática de esportes) e atividade sedentária (videogame, jogos de mesa, atividades culturais, televisão, etc.). Em seguida, questões sobre o quão ativo ou inativo o indivíduo se considera, indagando sobre práticas de atividades físicas, de leve a moderada, ou podendo também responder que não pratica nenhuma atividade. Nesse bloco também continha uma questão com a afirmativa "eu gosto de atividades físicas", na qual o avaliado tem que revelar se concorda ou descorda com a afirmação, e, por último, questões sobre o deslocamento para a escola e tempo de trajeto.

4) Tempo em frente à tela de TV: Neste bloco, as perguntas são referentes ao tempo em horas que o indivíduo assiste televisão, joga videogame e usa o computador durantes os dias de semana (que tem aula) e durante os finais de semana.

Os dados foram coletados no segundo semestre do ano de 2015, na unidade escolar, e contaram com o acompanhamento e supervisão de um membro efetivo da escola. No primeiro momento, os avaliadores entraram em contato com a direção da escola para esclarecer os objetivos e as propostas do projeto, quando se realizou também o sorteio dos alunos participantes. Após o sorteio, todos foram chamados pela diretora em uma sala separada para que os avaliadores explicassem a pesquisa, a interpretação de algumas questões e entregassem o TCLE para a autorização dos pais e/ou responsáveis.

Para análise estatística, os dados foram tabulados e categorizados no software Statistical Package for the Social Sciencies (SPSS), versão 21.0, avaliando a frequência absoluta e relativa, a média e o desvio padrão das variáveis estudadas. Para a comparação dos valores médios entre os sexos, foi utilizado o teste $t$ de Student para amostras independentes nas variáveis com distribuição normal. Para fazer a correlação entre os hábitos de atividades físicas e o comportamento sedentário, foi realizada a correlação "r" de Pearson. Todas as análises com nível de significância de $95 \%(p \leq 0,05)$.

A presente pesquisa recebeu aprovação do Comitê de Ética em Seres Humanos (CEP), da Universidade do Extremo Sul Catarinense (UNESC), sob Parecer $n^{\circ} .1 .125 .725 / 2015$.

\section{RESULTADOS}

Dos 86 adolescentes que participaram do estudo, a idade média apresentada foi de $16,57 \pm 1,07$ anos, sendo $52,3 \%$ do sexo feminino. A maior incidência foi de adolescentes do $3^{\circ}$ ano do ensino médio, num patamar de $45,3 \%$. Os adolescentes sem ocupação foram $67,4 \%$, e $84,9 \%$ se autodeclararam de cor branca (Tabela I). 
Tabela I - Caracterização da Amostra.

\begin{tabular}{lc}
\hline Características & Média/ D.P \\
\hline Idade & $16,57 \pm 1,07$ \\
Sexo & F.A. (F.R.\%) \\
$\quad$ Feminino & $45(52,3)$ \\
$\quad$ Masculino & $41(47,7)$ \\
Séries & \\
$1^{\circ}$ ano & $16(18,6)$ \\
$2^{\circ}$ ano & $31(36,0)$ \\
$3^{\circ}$ ano & $39(45,3)$ \\
Ocupação & \\
Sim & $28(32,6)$ \\
Não & $58(67,4)$ \\
Cor da pele & \\
Branca & $73(84,9)$ \\
Parda & $5(5,8)$ \\
Preta & $4(4,7)$ \\
Amarela & $3(3,5)$ \\
Indígena & $1(1,2)$ \\
\hline
\end{tabular}

Fonte: Dados da pesquisa; D.P: Desvio Padrão; FR: Frequência relativa; FA: Frequência Absoluta

$\mathrm{Na}$ Tabela II, referente aos hábitos alimentares dos adolescentes, pode-se verificar que $40,7 \%$ relataram que raramente ou quase nunca consomem uma dieta balanceada. Quanto à ingestão do café da manhã, 17,4\% responderam não o ter ingerido nos últimos 7 dias. Em relação ao consumo de bebidas alcoólicas nos últimos 30 dias, pode-se observar que $36 \%$ dos adolescentes relataram ter ingerido, no intervalo de 1 a 9 dias, 5 doses ou mais de bebidas alcoólicas em uma mesma ocasião.

Tabela II - Hábitos alimentares.

\begin{tabular}{lccc}
\hline Variáveis & & F.A. & F.R. (\%) \\
\hline & Quase nunca & 19 & 22,1 \\
Você consome uma dieta balanceada? & Raramente & 23 & 26,7 \\
& Algumas vezes & 29 & 33,7 \\
& Com relativa frequência & 9 & 10,5 \\
& Quase sempre & 6 & 7,0 \\
\hline Quantas vezes por dia você ingere bebidas que com cafeína? & Quase nunca & 9 & 10,5 \\
& Raramente & 26 & 30,2 \\
& Algumas vezes & 19 & 22,1 \\
Durante os últimos 7 dias, quantos dias você tomou café & Com relativa frequência & 24 & 27,9 \\
da manhã? & Quase sempre & 8 & 9,3 \\
\hline \multirow{2}{*}{ Durante os últimos 30 dias, quantos dias você tomou 5 doses } & Nenhum dia & 15,0 & 17,4 \\
ou mais de bebida alcoólica em uma mesma ocasião? & Até 3 dias & 19,0 & 22,1 \\
& De 4 a 7 dias & 52,0 & 60,5 \\
\hline
\end{tabular}

Fonte: Dados da pesquisa; D.P: Desvio Padrão; FR: Frequência relativa; FA: Frequência Absoluta 
Na Tabela III, sobre os hábitos de atividades físicas, pode-se observar que 53,5\% têm como lazer preferido as atividades físicas. Percebem-se vigorosamente ativos $57 \%$, e $51,2 \%$ concordam totalmente quanto ao gostar das atividades físicas. Quanto aos deslocamentos, 33,7\% deslocam-se a pé até a escola e 31,4\% utilizam a bicicleta.

Tabela III - Hábitos relacionados às atividades físicas.

\begin{tabular}{|c|c|c|c|}
\hline Variáveis & & F.A. & F.R. (\%) \\
\hline \multirow[t]{2}{*}{ Atividade de lazer preferida } & Atividades físicas & 46 & 53,5 \\
\hline & Atividades sedentárias & 39 & 45,3 \\
\hline \multirow{5}{*}{ Percepção do Nível de Atividade Física } & Sou vigorosamente ativo & 49 & 57,0 \\
\hline & Suficientemente ativo & 14 & 16,3 \\
\hline & Não sou, mas pretendo ser & 4 & 4,7 \\
\hline & Não sou, mas pretendo ser nos próximos 6 meses & 7 & 8,1 \\
\hline & Não sou, mas pretendo ser em menos de 6 meses & 12 & 14,0 \\
\hline \multirow{3}{*}{$\begin{array}{l}\text { Durante a semana quantos dias pratica } \\
\text { atividade vigorosa? }\end{array}$} & Nenhum dia & 11,0 & 12,8 \\
\hline & Até 3 dias & 42,0 & 48,8 \\
\hline & De 4 a 7 dias & 33,0 & 38,4 \\
\hline \multirow{3}{*}{$\begin{array}{l}\text { Quantas vezes na semana você é } \\
\text { vigorosamente ativo pelo menos } 30 \text { minutos } \\
\text { por dia? }\end{array}$} & Menos que uma vez & 21,0 & 24,4 \\
\hline & 1 a 3 vezes & 37,0 & 43,0 \\
\hline & 4 vezes ou mais & 27,0 & 31,4 \\
\hline \multirow{3}{*}{$\begin{array}{l}\text { Nos últimos } 7 \text { dias quantos dias você foi } \\
\text { fisicamente ativo por } 60 \text { minutos? }\end{array}$} & Nenhum dia & 12,0 & 14,0 \\
\hline & Até 3 dias & 48,0 & 55,8 \\
\hline & De 4 a 7 dias & 26,0 & 30,2 \\
\hline \multirow{5}{*}{ Gosto por atividade física } & Discordo totalmente & 3 & 3,5 \\
\hline & Concordo em parte & 27 & 31,4 \\
\hline & Discordo em parte & 1 & 1,2 \\
\hline & Concordo totalmente & 44 & 51,2 \\
\hline & Nem concordo nem discordo & 8 & 9,3 \\
\hline \multirow{4}{*}{ Deslocamento à escola } & A pé & 29 & 33,7 \\
\hline & Bicicleta & 27 & 31,4 \\
\hline & Carro/ Moto & 7 & 8,1 \\
\hline & Ônibus & 23 & 26,7 \\
\hline \multirow{6}{*}{ Tempo de deslocamento } & $<10 \min$ & 48 & 55,8 \\
\hline & 10- 19 & 24 & 27,9 \\
\hline & $20-29$ & 8 & 9,3 \\
\hline & 30- 39 & 2 & 2,3 \\
\hline & 40- 49 & 1 & 1,2 \\
\hline & $<60 \min$ & 3 & 3,5 \\
\hline
\end{tabular}

Fonte: Dados da pesquisa; D.P: Desvio Padrão; FR: Frequência relativa; FA: Frequência Absoluta

Tabela IV - Tempo em frente à tela.

\begin{tabular}{|c|c|c|c|}
\hline Variáveis & Sexo & Média & D.P \\
\hline \multirow{2}{*}{ Quantas horas por dia você assiste TV nos dias de aulas? } & Masculino & 93,6 & 101,2 \\
\hline & Feminino & $132,2^{*}$ & 153,5 \\
\hline \multirow{2}{*}{ Quantas horas por dia assiste TV nos finais de semana? } & Masculino & 198,5 & 174,4 \\
\hline & Feminino & 156,4 & 134,5 \\
\hline \multirow{2}{*}{ Quantas horas por dia você usa computador nos dias de aula? } & Masculino & $303,5^{*}$ & 443,1 \\
\hline & Feminino & 187,3 & 175,4 \\
\hline \multirow{2}{*}{ Quantas horas por dia você usa computador nos finais de semana? } & Masculino & $320,7^{*}$ & 321,4 \\
\hline & Feminino & 191,1 & 250,4 \\
\hline \multirow{2}{*}{ Quantas horas por dia você joga videogame nos dias de aula? } & Masculino & $78,3^{*}$ & 144,2 \\
\hline & Feminino & 4,9 & 25,3 \\
\hline \multirow{2}{*}{ Quantas horas por dia você joga videogame nos finais de semana? } & Masculino & $103,2^{*}$ & 233,0 \\
\hline & Feminino & 9,1 & 36,7 \\
\hline
\end{tabular}

Fonte: Dados da pesquisa; D.P: Desvio Padrão 
Ao comparar o sexo masculino com o sexo feminino quanto ao tempo em frente à tela de TV nos dias de aula e nos finais de semana, pôde-se verificar que há um comportamento diferente entre os sexos. Os participantes do sexo masculino demostraram passar mais tempo em comportamentos sedentários, como em frente à televisão, computadores e videogames, tanto nos dias de semana quanto nos finais de semana (Tabela IV).

Nos dados de correlação (Tabela $V$ ) entre hábitos de atividades físicas e lazer preferido, verificam-se correlações negativas $(r=-0,257$ e $r=-0,022)$ para atividades físicas vigorosas por 30 minutos durante a semana e atividades físicas por 60 minutos nos últimos sete dias, respectivamente. Quanto ao tempo em frente à tela nos dias da semana e finais de semana, foi possível observar uma correlação positiva com atividades físicas vigorosas, porém não houve significância estatística $(p>0,05)$.

Tabela V - Correlação entre as variáveis da atividade física e comportamentos sedentários.

\begin{tabular}{lcccccc}
\hline Variáveis & $\mathbf{( 1 )}$ & $\mathbf{( 2 )}$ & $\mathbf{( 3 )}$ & $\mathbf{( 4 )}$ & $\mathbf{( 5 )}$ & $\mathbf{( 6 )}$ \\
\hline Dias da semana que faz atividades físicas vigorosas por 30' (1) & 1 & $0,774^{* *}$ & $-0,257^{*}$ & 0,028 & 0,178 & $-0,172$ \\
Nos últimos 7 dias, foi fisicamente ativo por 60' (2) & $0,774^{* *}$ & 1 & $-0,022$ & $-0,099$ & 0,119 & 0,005 \\
Lazer preferido (3) & $-0,257^{*}$ & $-0,022$ & 1 & $-0,096$ & $-0,120$ & 0,184 \\
Tempo de tela - na semana (4) & 0,028 & $-0,099$ & $-0,096$ & 1 & $0,358^{* *}$ & 0,054 \\
Tempo de tela - aos fins de semana (5) & 0,178 & 0,119 & $-0,120$ & $0,358^{* *}$ & 1 & 0,036 \\
Deslocamento ativo (6) & $-0,172$ & 0,005 & 0,184 & 0,054 & 0,036 & 1 \\
\hline
\end{tabular}

Fonte: Dados da pesquisa; $\left({ }^{*}\right)$ A correlação é significativa no nível de $0,01 .\left({ }^{* *}\right)$ A correlação é significativa no nível de 0,05

\section{DISCUSSÃO}

O presente estudo identificou diferentes comportamentos de risco à saúde entre adolescentes do ensino médio da rede pública do município de Praia Grande - Santa Catarina. Os dados sociodemográficos são semelhantes aos encontrados em estudo prévio da região, que avaliaram a prevalência de comportamentos de risco, os hábitos alimentares e a satisfação corporal dos adolescentes ${ }^{(13)}$.

Verificou-se que grande parte da amostra estudada raramente ou quase nunca consome uma dieta balanceada. São dados preocupantes, uma vez que a alimentação adequada e saudável, quando praticada de maneira regular na adolescência, é um fator determinante para o desenvolvimento e manutenção do organismo, além de contribuir para a prevenção de diferentes tipos de doenças cardiometabólicas, como a hipercolesterolemia, a hipertensão arterial, a diabetes tipo 2, as doenças esqueléticas, além de vários tipos de câncer e distúrbios alimentares ${ }^{(14)}$. Estudos têm relacionado o surgimento desses agravos ao sobrepeso e obesidade, considerados como problemas de saúde de maior crescimento dentre as doenças crônicas não transmissíveis em adolescentes ${ }^{(15-16)}$.

A omissão do café da manhã foi reportada por quase $20 \%$ dos adolescentes avaliados, semelhante a outro estudo transversal, nacional e de base escolar ${ }^{(17)}$, que encontrou uma prevalência de $21,9 \%$ na omissão do café da manhã junto a adolescentes brasileiros. Um estudo transversal, de base populacional, realizado com adolescentes do município de São Paulo, São Paulo, Brasil, encontrou resultados superiores, de $38 \%{ }^{(18)}$. A omissão de refeições é um hábito muito comum entre os adolescentes, especialmente o café da manhã. Essa omissão pode ser prejudicial, pois indivíduos que consomem o café da manhã, quando comparados aos indivíduos que omitem essa refeição, têm maior aporte de micronutrientes e tendem a alcançar as recomendações nutricionais ${ }^{(18)}$.

Em relação ao consumo de bebidas alcoólicas nos últimos 30 dias, pôde-se observar que boa parte dos adolescentes relatou ingerir de 1 a 9 dias, 5 doses ou mais de bebidas alcoólicas em uma mesma ocasião, o que é considerado um consumo excessivo. Esses resultados são superiores aos encontrados no estudo transversal realizado com adolescentes escolares de Porto Velho, Rondônia, cuja prevalência do consumo de bebidas alcoólicas nos últimos 30 dias foi de $24 \%{ }^{(19)}$.

A crença de que a bebida alcóolica não é droga contribui para o estímulo e incentivo ao seu uso, principalmente entre os adolescentes ${ }^{(20)}$. Isto acontece pelo fato do álcool ser facilmente obtido e fartamente propagandeado, refletindo em um consumo precoce e disseminado. Esse cenário aponta para uma recorrente infringência do ordenamento jurídico vigente, já que a lei brasileira (Lei $n^{\circ}$ 9294/1996) proíbe a venda de bebidas alcoólicas para menores de 18 anos $^{(21)}$.

Todavia a preocupação com a ingestão de bebidas alcóolicas na adolescência transcende o ordenamento jurídico, pois está associada a uma série de comportamentos de risco, além de aumentar a chance de envolvimento em 
acidentes, violência sexual, participação em gangues, queda no desempenho escolar, dificuldades de aprendizado, prejuízo no desenvolvimento e estruturação das habilidades cognitivo-comportamentais e emocionais do jovem ${ }^{(21)}$. Aponta-se ainda a associação do uso de álcool com a experimentação de outras drogas ${ }^{(22)}$.

Quanto aos hábitos de atividades físicas, pôde-se observar que mais da metade dos adolescentes as têm como lazer preferido, $57 \%$ percebem-se vigorosamente ativos e $51,2 \%$ concordam totalmente quanto ao gostar da prática, diferente do encontrado em pesquisas recentes, nas quais fica evidenciado os baixos níveis de atividade física entre os adolescentes brasileiros ${ }^{(8)}$. Esses resultados podem ser justificados pelo fato de se tratar de um município pequeno, com baixos índices de criminalidade, com predomínio das atividades agrícolas, proporcionando aos jovens uma maior liberdade pública para a prática de atividades físicas ao ar livre, além de auxiliar nas atividades agrícolas da família no contra turno escolar.

Quanto aos deslocamentos, boa parte desloca-se a pé à escola ou utiliza a bicicleta. Um estudo realizado com 741 adolescentes escolares, com idade entre 11 a 18 anos, de escolas públicas e privadas de Curitiba, Paraná, encontrou uma prevalência de $42,9 \%$ (50\% meninos e $37,2 \%$ meninas) de deslocamento ativo para a escola(23). 0 deslocamento ativo tem sido um dos principais domínios da atividade física utilizados em estratégias de intervenção para um estilo de vida ativo em crianças e adolescentes, pois é um comportamento que possibilita a adoção de um hábito diário na rotina dos jovens ${ }^{(24)}$.

O tempo de deslocamento gasto encontrado no presente estudo é baixo, com maior prevalência de 10 a 19 minutos, característica de municípios de pequeno porte, com proximidade entre casa e escola. Apesar do tempo gasto com o deslocamento ativo ser abaixo do tempo preconizado pelas últimas recomendações de atividades físicas para crianças e adolescentes ${ }^{(25)}$, contribui para o aumento do volume total de atividades físicas, favorecendo na melhora dos níveis de saúde ${ }^{(24)}$ e na diminuição da incidência de sobrepeso e obesidade nesse público ${ }^{(11)}$.

Quando comparados o tempo em frente à tela entre adolescentes do sexo masculino e do sexo feminino, pôde-se verificar que há uma diferenciação entre os sexos. Os meninos demonstraram passar mais tempo em comportamentos sedentários (em frente à televisão, computadores e videogames), tanto em dias de semana quanto aos finais de semana, quando comparados com as meninas. Recomenda-se que crianças e adolescentes limitem a, no máximo, duas horas por dia o tempo dedicado às atividades em frente à tela ${ }^{(25)}$. No presente estudo, a média do tempo é praticamente três vezes maior que a indicada. Crianças e adolescentes expostos a um tempo maior que duas horas diárias em frente à tela possuem maior pré-disposição a problemas de saúde, como hipertensão arterial, síndrome metabólica, obesidade, entre outros ${ }^{(26)}$

Ao verificar a correlação entre hábitos de atividade física e lazer preferido, observou-se no presente estudo uma correlação negativa $(r=-0,257$ e $r=-0,022)$ para atividades físicas vigorosas por 30 minutos durante a semana e atividades físicas por 60 minutos nos últimos sete dias, respectivamente, indicando que a atividade física não faz parte do lazer preferido desses sujeitos. Em relação ao tempo em frente à tela nos dias de semana e finais de semana, a correlação foi positiva com atividades físicas vigorosas, porém não foi significativamente estatística $(p>0,05)$, indicando que o tempo em frente à tela não interfere nos níveis de atividade física, muito provavelmente em razão das características que o município apresenta, com predomínio das atividades agrícolas.

É importante destacar que, para que haja efetivas mudanças frente a esses comportamentos, faz-se necessário incluir nessas estratégias os pais, os familiares e os pares, por serem considerados preponderantes para a adoção de hábitos saudáveis de vida. Sugere-se, ainda, que os professores de Educação Física desenvolvam conteúdos relacionados aos hábitos saudáveis, direcionando-os para a promoção da saúde e a prevenção de doenças, o que poderá contribuir para uma saúde coletiva mais efetiva.

\section{CONCLUSÃO}

Conclui-se que as características geográficas, as atividades físicas habituais dos adolescentes e a segurança pública contribuem para um estilo de vida mais ativo fora do turno escolar, mas não minimizam hábitos incorretos cotidianamente encontrados em grandes centros urbanos.

\section{CONFLITOS DE INTERESSE}

Não houve conflitos de interesse no presente estudo.

\section{REFERÊNCIAS}

1. Sousa ZAA, Silva JG, Ferreira MA. Saberes e práticas de adolescentes sobre saúde: implicações para o estilo 
de vida e cuidado de si. Esc Anna Nery Rev Enferm. 2014;18(3):400-6.

2 Silva LVM, Packe C, Tkac C, Braga RK, Urbinati KS. Associação entre variáveis de composição corporal e aptidão física em adolescentes. Rev Aten Saúde. 2014;12(41):51-6.

3. Vitorino PVO, Barbosa MA, Sousa ALL, Jardim PCBV, Ferreira SS. Prevalência de estilo de vida sedentário entre adolescentes. Acta Paul Enferm. 2014;27(1):166-71.

4. Farias JC Jr, Reis RS, Hallal PC. Physical activity, psychosocial and perceived environmental factors in adolescents from Northeast Brazil. Cad Saúde Pública. 2014;30(5):941-51.

5. Cureau FV, Silva TLN, Bloch KV, Fujimori E, Belfort DR, Carvalho KMB, et al. ERICA: inatividade física no lazer em adolescentes brasileiros. Rev Saúde Pública. 2016;50(Supl 1):1s-11s.

6. Alwan A. Global status report on noncommunicable diseases 2010. Geneva: World Health Organization; 2011.

7. Brito ALS, Hardman CM, Barros MVG. Prevalência e fatores associados à simultaneidade de comportamentos de risco à saúde em adolescentes. Rev Paul Pediatr. 2015;33(4):423-30.

8. Instituto Brasileiro de Geografia e Estatística, Coordenação de População e Indicadores Sociais. Pesquisa Nacional de Saúde do Escolar: 2015. Rio de Janeiro: IBGE; 2016.

9. Louzada MLC. Nutrição e saúde: o papel do ultraprocessamento de alimentos [tese]. São Paulo: Universidade de São Paulo; 2015.

10. Azeredo CM, Rezende LFM, Canella DS, Claro RM, Castro IRR, Luiz OC, et al. Dietary intake of Brazilian adolescents. Public Health Nutr. 2015;18(7):1215-24.

11. Bezerra IN, Gurgel AOC, Barbosa RGB, Silva GB Jr. Dietary behaviors among young and older adults in Brazil. J Nutr Health Aging. 2018;22(5):575-80.

12. Instituto Brasileiro de Geografia e Estatística. Censo Demográfico 2010. Rio de Janeiro: IBGE; 2011.

13. Pereira EV, Antunes RC, Viero VSF, Volpato AMJ, Faras JM. Prevalência de comportamentos de risco, hábitos alimentares e satisfação corporal dos adolescentes de Criciúma/SC. In: Conselho Regional de Educação Física de Santa Catarina. Boas práticas na educação física catarinense 2017. 3rd ed. Florianopolis: CREF3/SC; 2017. p. 95-111.

14. Assis MM, Penna LF, Neves CM, Mendes APCC, Oliveira RMS, Netto MP. Avaliação do conhecimento nutricional e comportamento alimentar após educação alimentar e nutricional em adolescentes de Juiz de Fora - MG. HU Rev. 2014;40(3-4):135-43.

15. Carneiro CS, Peixoto MRG, Mendonça KL, Póvoa TIR, Nascente FMN, Jardim TSV, et al. Excesso de peso e fatores associados em adolescentes de uma capital brasileira. Rev Bras Epidemiol. 2017;20(2):260-73.

16. Rocha NP, Milagres LC, Longo GZ, Ribeiro AQ, Novaes JF. Associação entre padrão alimentar e risco cardiometabólico em crianças e adolescentes: uma revisão sistemática. J Pediatr. 2017;93(3):214-22.

17. Barufaldi LA, Abreu GA, Oliveira JS, Santos DF, Fujimori E, Vasconcelos SML, et al. ERICA: prevalência de comportamentos alimentares saudáveis em adolescentes brasileiros. Rev Saúde Pública. 2016;50(supl 1):1s9s.

18. Marchioni DML, Gorgulho BM, Teixeira JA, Verly E Jr, Fisberg RM. Prevalência de omissão do café da manhã e seus fatores associados em adolescentes de São Paulo: estudo ISA-Capital. Nutrire. 2015;40(1):10-20.

19. Elicker E, Palazzo LS, Aerts DRGC, Alves GG, Câmara S. Uso de álcool, tabaco e outras drogas por adolescentes escolares de Porto Velho-RO, Brasil. Epidemiol Serv Saude. 2015;24(3):399-410.

20. Zeitoune RCG, Ferreira VS, Silveira HS, Domingos AM, Maia AC. O conhecimento de adolescentes sobre drogas lícitas e ilícitas: uma contribuição para a enfermagem comunitária. Esc Anna Nery Rev Enferm. 2012;16(1):5763.

21. Brasil. Lei $n^{\circ} 9.294$, de 15 de Julho de 1996. Dispõe sobre as restrições ao uso e à propaganda de produtos fumígeros, bebidas alcoólicas, medicamentos, terapias e defensivos agrícolas, nos termos do $\S 4^{\circ}$ do art. 220 da Constituição Federal. Diário Oficial da União; Brasília, 15 Jul. 1996; Seção 1. 
22. Malta DC, Machado IE, Porto DL, Silva MMA, Freitas PC, Costa AWN, et al. Consumo de álcool entre adolescentes brasileiros segundo a Pesquisa Nacional de Saúde Escolar (PeNSE 2012). Rev Bras Epidemiol. 2014;17(supl 1):203-14.

23. Becker LA, Fermino RC, Lima AV, Rech CR, Añez CRR, Reis RS. Perceived barriers for active commuting to school among adolescents from Curitiba, Brazil. Rev Bras Ativ Fís Saúde. 2017;22(1):24-34.

24. Del Duca GF, Barros MVG, Silva KS, Garcia LMT, Bezerra J, Nahas MV. Effectiveness on physical activity indicators of an intervention delivered to high school students. Rev Bras Cineantropom Desempenho Hum. 2014;16(supl 1):13-24.

25. Council on Communications and Media. Children, adolescents, and the media. Pediatrics. 2013;132(5):958- 61.

26. Friedrich RR, Polet JP, Schuch I, Wagner MB. Efeito dos programas de intervenção no âmbito escolar para reduzir o tempo gasto em frente a telas: uma meta-análise. J Pediatr. 2014;90(3):232-41.

\section{Endereço para correspondência:}

Francielle Klouck

Universidade do Extremo Sul Catarinense - UNESC

Departamento do Curso de Educação Física

Av. Universitária, 1105

Bairro: Universitário

CEP: 88806-000 - Criciúma - SC - Brasil

E-mail: vanisedossantos@hotmail.com/jmf@unesc.net 